\title{
Germinação de sementes osmocondicionadas e não osmocondicionadas e crescimento inicial de Physalis angulata L. (Solanaceae) em ambientes salinos
}

\author{
Manuela Oliveira de Souza ${ }^{1,2}$, Cíntia Luiza Mascarenhas de Souza ${ }^{1}$ e Claudinéia Regina Pelacani ${ }^{1}$
}

Recebido em 18/05/2010. Aceito em 15/12/2010

\begin{abstract}
RESUMO
(Germinação de sementes osmocondicionadas e não osmocondicionadas e crescimento inicial de Physalis angulata L. (Solanaceae) em ambientes salinos). O gênero Physalis pertencente à família Solanaceae, possui 110 espécies, das quais 11 ocorrem no Brasil. Physalis angulata L. destaca-se no gênero pela presença de vitaesteróides, principalmente as fisalinas, encontradas em raízes, caules e folhas, com propriedades imunomoduladora, antimicrobiana e anticancerígena. Este trabalho teve por objetivo avaliar a germinabilidade de sementes osmocondicionadas e não osmocondicionadas e o crescimento inicial de $P$. angulata em ambientes salinos. Foram realizados experimentos nos quais as sementes foram submetidas ao osmocondicionamento (PEG 6000) e colocadas para germinar em soluções

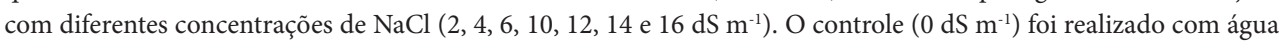
destilada. Os parâmetros avaliados foram a germinabilidade das sementes, o índice de velocidade de germinação das sementes (IVG), o coeficiente de uniformidade de germinação e a massa seca total das plântulas. Houve diminuição da germinabilidade e do IVG, em ambos os tratamentos. No entanto, as sementes osmocondicionadas apresentaram maior tolerância ao estresse salino. Apesar da redução significativa da massa seca em relação ao aumento da salinidade do meio, o osmocondicionamento como tratamento pré-germinativo poderia ser utilizado para favorecer a tolerância ao estresse salino, uma vez que a germinabilidade foi maior para sementes osmocondicionadas.
\end{abstract}

Palavras-chave: $\mathrm{NaCl}$, massa seca, $\mathrm{PEG} 6000$, salinidade, taxa de germinação

\begin{abstract}
(Germination of osmoprimed and non-osmoprimed seeds and initial growth of Physalis angulata (Solanaceae) in saline environments). The genus Physalis belongs to the Solanaceae family, with 110 species, of which 11 occur in Brazil. Physalis angulata L. stands out in the genus due to the presence of vitasteroids, especially physalins, found in roots, stems and leaves, showing antimicrobial, anticancer and immunomodulatory properties. This study aimed to evaluate the germination of osmotic priming treatment and non-primed seeds and the initial growth of P. angulata in saline environments. The seeds were subjected to priming (PEG 6000) and incubated in solutions with increasing concentration of $\mathrm{NaCl}\left(2,4,6,10,12,14\right.$ and $\left.16 \mathrm{dS} \mathrm{m}^{-1}\right)$. Distilled water was used as control $\left(0 \mathrm{dS} \mathrm{m}^{-1}\right)$. The parameters evaluated were seed germinability, rate of germination, coefficient of uniformity and total dry mass of seedlings. A decrease in germinability and IVG was observed in both treatments. However, primed seeds showed higher tolerance of salt stress. Despite the significant reduction in dry mass with increasing salinity, priming as a pre-germinative treatment could be used to foster tolerance, since germinability was higher for primed seeds.
\end{abstract}

Key words: $\mathrm{NaCl}$, dry mass, PEG 6000, salinity, rate of germination

\section{Introdução}

Physalis pertencente à família Solanaceae, inclui 110 espécies e ocupa lugar de destaque na família pela presença de metabólitos poli-oxigenados e vitaesteróides (Tomassini et al. 2000), substâncias às quais se atribuem várias atividades farmacológicas. O gênero é de fácil reconhecimento, especialmente pelo tipo de fruto, uma baga com o cálice persistente. Para o Brasil, são reconhecidas 11 espécies (D`Arcy et al. 2005), com distribuição nas várias regiões do país, especialmente na Amazônia e no Nordeste, onde são encontradas 6 espécies.

Physalis angulata L., também conhecida como camapú, mullaca ou juá de capote pode ser encontrada em quase todo

1 Universidade Estadual de Feira de Santana, Feira de Santana, BA, Brasil

2 Autora para correspondência: oliva.manuela@gmail.com 
território brasileiro. Na Bahia, existem registros em Seabra, Água Quente, Mucugê e Rio de Contas (Matos 2000). P. angulata tem sido utilizada pela medicina popular como anticoagulante, antileucêmico, antimutagênico, antiinflamatório, antiespasmódico, antiséptico, analgésico e no tratamento de diabetes, entre outros (Lin et al. 1992; Chiang et al. 1992). Além de ser amplamente utilizada na medicina popular, esta espécie tem despertado grande interesse tanto pelo teor de fisalinas quanto por suas propriedades farmacológicas.

Nas regiões áridas e semi-áridas, o excesso de sais no solo é um fator limitante na produção agrícola. No Brasil, estas áreas estão localizadas principalmente no semi-árido nordestino, local de grande ocorrência da espécie em estudo. Em geral, a salinização afeta negativamente não só a germinação, como o desenvolvimento da planta e a produtividade. Em casos mais extremos causa a deformidade e a morte das plantas (Silva \& Pruski 1997). No entanto, os mecanismos de tolerância à salinidade nos vegetais ainda não estão completamente esclarecidos pelo fato de envolver alterações bioquímicas, fisiológicas e moleculares (Zhu 2002; Abreu et al. 2008).

A tolerância ao estresse salino varia de acordo com a espécie. Um dos mecanismos citados que proporcionam maior resistência a ambientes salinos é a capacidade das plantas em acumular íons nos vacúolos, ou ainda solutos orgânicos de baixo peso molecular, capazes de manter a absorção de água e a turgescência celular (Munns \& Tester 2008).

O condicionamento osmótico "priming" consiste num pré-tratamento, no qual as sementes são imersas em solução osmótica sob tempo e temperatura determinados de modo a restringir a quantidade de água absorvida (Anwar et al. 1978; Wien 1997). Considerando o padrão trifásico, ocorrem as fases iniciais da germinação (fase I e II), com os eventos pré-metabólicos germinativos, sem ocorrer o estádio de emergência da radícula (fase III) (Bradford 1986; Bewley \& Black 1994). Assim, quando semeadas, a emergência das plântulas será rápida, sincronizada e em maior porcentagem. As respostas ao condicionamento osmótico variam entre as espécies, e mesmo entre os lotes de sementes de uma mesma espécie (Brocklehurst \& Dearman 1983; Nascimento 1998).

A solução de polietilenoglicol (PEG) tem sido usada como meio osmótico para simular o estresse hídrico que poderia induzir a dormência secundária. Porém é necessário que a semente alcance determinado nível de umidade, cujo teor é variável com a espécie (Borges et al. 2002). Diversos benefícios têm sido relatados com o emprego do osmocondicionamento, dentre eles, a maior probabilidade de se obter uma melhor germinação e emergência, particularmente em condições de estresse (Warren \& Bennett 1997; Mohammadi 2009).

Compreender como estes eventos influenciam no desenvolvimento de Physalis angulata torna-se importante para os ajustes nos protocolos de propagação da espécie, principalmente quando se analisa o efeito de tratamentos pré-germinativos, como é o caso do osmocondicionamento na germinação e desenvolvimento da plântula frente ao estresse salino.

Assim, o presente trabalho teve como objetivo avaliar a germinabilidade de sementes osmocondicionadas e o crescimento inicial de Physalis angulata L. em ambientes salinos.

\section{Material e métodos}

Os frutos maduros de Physalis angulata foram coletados manualmente em janeiro de 2009, no Horto Florestal da Universidade Estadual de Feira de Santana. As sementes foram retiradas sob água corrente atéa separação completa da polpa. Estas foram colocadas para secar sobre papel filtro em temperatura ambiente (condições de laboratório) e foram armazenadas, sob as mesmas condições, até o momento dos ensaios.

Foram utilizadas sementes de Physalis angulata em duas condições: osmocondicionadas e não osmocondicionadas. As sementes foram condicionadas em solução aerada de polietilenoglicol (PEG 6000; Vilela et al. 1991) apresentando potencial osmótico de -1,2 MPa, previamente estabelecido como potencial de priming da espécie (Souza 2009). As amostras de sementes ( 1.000 sementes) de $P$. angulata foram colocadas em tubo de ensaio, contendo $25 \mathrm{ml}$ de solução osmótica, conectado a um sistema de aeração artificial (bomba de aquário). As sementes foram condicionadas nesta solução durante 10 dias e mantidas em câmara de germinação (Eletrolab) a $35^{\circ} \mathrm{C}$, ajustada para um fotoperíodo de 12 horas (Souza 2009). A solução de PEG 6000 foi trocada a cada dois dias e no $10^{\circ} \mathrm{dia}$, as sementes foram retiradas da solução e colocadas para secar em temperatura ambiente, por 24 horas para atingir o peso inicial.

Para os experimentos de germinação, as sementes de Physalis angulata osmocondicionadas (OS) e não osmocondicionadas (NO) foram dispostas em placas de Petri de vidro $(6 \times 6 \mathrm{~cm})$, contendo ao fundo duas folhas de papel germitest (semi-esterilizados em estufa de secagem a $105^{\circ} \mathrm{C}$, por 4 horas), umedecidas com $3 \mathrm{ml}$ de água destilada (controle) e diferentes concentrações de $\mathrm{NaCl}(2,4,6,10,12$, 14 e $16 \mathrm{dS} \mathrm{m}^{-1}$ ). As concentrações de cada solução foram aferidas quanto à condutividade elétrica, em aparelho Bel Engineering, modelo W 12-D. As sementes foram acondicionadas em câmara de germinação (Eletrolab) a $35^{\circ} \mathrm{C}$, ajustada para um fotoperíodo de 12 horas, por 10 dias. Cada tratamento foi constituído de quatro repetições com 25 sementes cada. As avaliações foram realizadas diariamente e foram consideradas germinadas as sementes que emitiram a radícula. As sementes foram transferidas para placa de Petri maior $(9,5 \times 9,5 \mathrm{~cm})$ nas mesmas condições descritas acima, por 10 dias para análise do crescimento.

Os parâmetros avaliados foram taxa de germinabilidade das sementes (\%), freqüência relativa (\%), índice de velocidade de germinação das sementes (IVG, sem.dias ${ }^{-1}$ ) e coeficiente de uniformidade de germinação (GUG, sem. $\operatorname{dias}^{-2}$ ) e massa seca total das plântulas (mg/plântula). 
As plântulas com 20 dias após a germinação foram colocadas em estufa de ventilação forçada a $40^{\circ} \mathrm{C}$ por 5 dias. Após esse período, as amostras foram armazenadas em dessecador com sílica, e posteriormente pesadas em balança de precisão de $0,0001 \mathrm{~g}$. O peso da massa seca por repetição foi determinado e expresso em $\mathrm{mg} /$ plântula (Nakagawa 1999).

O delineamento experimental utilizado foi inteiramente ao acaso. Os dados de germinação e massa seca foram submetidos à análise de variância pelo teste $\mathrm{F}$ e ajustaramse equações de regressão como variáveis dependentes do nível de salinidade. Os dados de velocidade média, IVG e CUG foram submetidos à análise de variância e para comparação entre as médias utilizou-se o teste de Tukey a $5 \%$ de probabilidade. A análise dos dados foi realizada por meio do programa computacional SISVAR (Ferreira 2000).

\section{Resultados e discussão}

Os dados de germinabilidade demonstram uma tendência quadrática negativa, havendo um decréscimo da porcentagem de germinação de sementes de Physalis angulata osmocondicionadas e não osmocondicionadas, à medida que aumentou a concentração das soluções salinas (Fig. 1).

A partir da concentração com CE de $12 \mathrm{dS} \mathrm{m}^{-1}$, as sementes não osmocondicionadas apresentaram taxa de ger- minação de $18 \%$, enquanto as sementes osmocondicionadas tiveram $83 \%$ das sementes germinadas. Nas duas maiores concentrações salinas, CEs $14 \mathrm{e} 16 \mathrm{dS} \mathrm{m}^{-1}$, as porcentagens de germinação foram, respectivamente de $52 \%$ e $41 \%$, para sementes osmocondicionadas e $4 \%$ e $7 \%$ para sementes não osmocondicionadas. O osmocondicionamento parece proporcionar maior resistência ao estresse salino em sementes de Physalis angulata.

A redução das taxas de germinação das sementes pode ser explicado pelo efeito osmótico da solução salina sobre a absorção de água pelas sementes, bem como um provável efeito tóxico do $\mathrm{NaCl}$ no embrião, principalmente em sementes não osmocondicionadas. Os sais de alta solubilidade, como o $\mathrm{NaCl}$, são os mais nocivos, pois as sementes ao absorverem água do substrato, absorvem também os sais que em excesso, provocam toxidez, acarretando distúrbios fisiológicos e diminuição no potencial germinativo (Ferreira 1997). O aumento do teor de sais no substrato provoca redução do potencial hídrico, induzindo menor capacidade de absorção de água pelas sementes, com influência direta na germinação e no desenvolvimento das plântulas (Rebouças et al. 1989; Ashraf \& Foolad, 2005; Flors et al. 2007).

Os diversos efeitos causados em decorrência das altas concentrações dos íons $\mathrm{Na}^{+}$e $\mathrm{Cl}^{-}$estão relacionados ao intumescimento do protoplasma, promovendo alterações no metabolismo, como diminuição de energia e distúrbios na assimilação de nitrogênio. Estas alterações refletem dire-

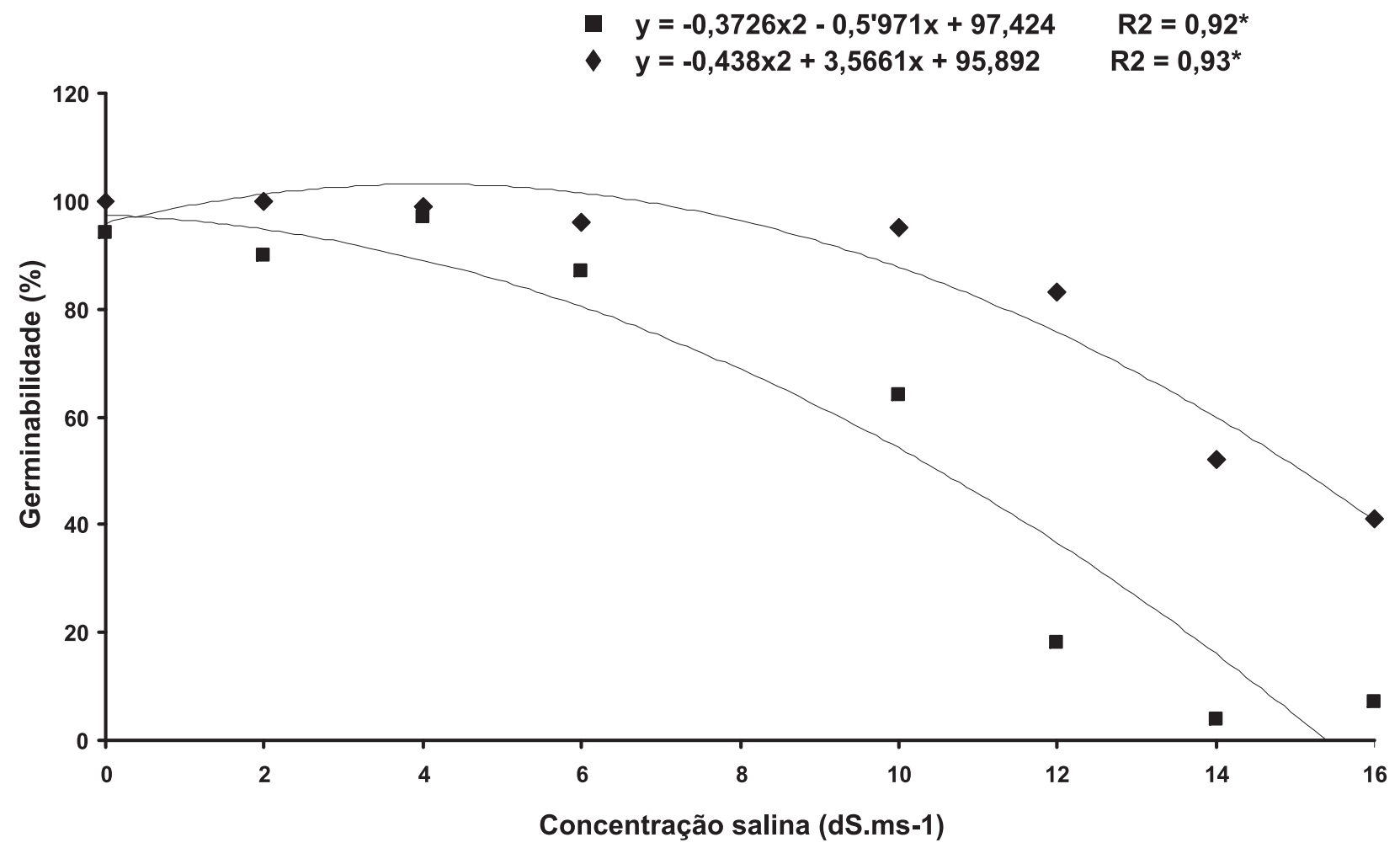

Figura 1. Germinabilidade de sementes de Physalis angulata L. oriundas de sementes osmocondicionadas (OS) e não osmocondicionadas (NO). OS ( ) e NO ( $\mathbf{\square})$ * Significativo a $5 \%$ de probabilidade. 
tamente na produção de proteínas, principalmente aquelas que compõem membranas, além de enzimas específicas envolvidas no processo germinativo (Larcher 2000).

Os mecanismos de tolerância das sementes submetidas à salinidade dependem da habilidade do protoplasma na compartimentalização dos íons que entram na célula. A maior parte dos íons é acumulada nos vacúolos e são diversas as modificações no metabolismo da planta induzidas pela salinidade. Entre estas destacam-se as modificações no balanço iônico, o comportamento estomático e eficiência fotossintética, o déficit hídrico e o desequilíbrio nutricional (Heuer 1997). Outro mecanismo para reduzir os efeitos tóxicos do $\mathrm{Na}^{+}$e $\mathrm{Cl}^{-}$é a redução da transpiração que leva a interrupção do fluxo xilemático para minimizar a entrada de sódio e cloro na planta (Flors et al. 2007).

O índice de velocidade de germinação (IVG) também mostrou um aumento em sementes osmocondicionadas de Physalis angulata (Tab. 1). É possível observar que o IVG para as sementes osmocondicionadas manteve-se com valores semelhantes ao controle $(7,2)$, até a concentração salina com CE $10 \mathrm{dS} \mathrm{m}^{-1}$. A partir desta CE houve um decréscimo significativo no IVG nas soluções salinas com CEs 12, 14 e $16 \mathrm{dS} \mathrm{m}^{-1}$. Estes resultados sugerem que as sementes tratadas com solução de $\mathrm{PEG}$ podem ter passado por um processo de adaptação à salinidade, pelo menos até a concentração salina com CE de $10 \mathrm{ds} . \mathrm{m}^{-1}$, como um processo de osmorregulação, que pode ser induzido por solutos orgânicos como açúcares, ácidos orgânicos, aminoácidos livres e prolina, evitando também a desidratação (Queiroga et al. 2006). Já para as sementes não osmocondicionadas, além da diminuição progressiva da taxa de germinação (Fig. 1), foi possível também observar que o IVG sofreu decréscimo com o aumento da concentração salina (CE de $6 \mathrm{dS} \mathrm{m}^{-1}$ ). Este índice foi ainda menor nas concentrações salinas mais elevadas, com diminuição de até 0,3 para a solução salina com CE de $16 \mathrm{dS} \mathrm{m}^{-1}$.

Os polígonos de freqüência relativa obtidos para a germinação de sementes de Physalis angulata são mostrados na Fig.
2. Observou-se que os polígonos obtidos tanto no controle como nas diferentes concentrações salinas apresentaram-se unimoidais, principalmente para sementes osmocondicionadas. No entanto, em concentração salina com CE $6 \mathrm{dS} \mathrm{m}^{-1}$, observou-se um polígono polimoidal para sementes não osmocondicionadas. Esses resultados refletem uma maior uniformidade da germinação, corroborada pelos coeficientes de uniformidade obtidos (Tab. 1). Não houve diferença significativa para as sementes osmocondicionadas e não osmocondicionadas em quaisquer das soluções testadas quando se compara os CUGs entre as soluções salinas de um mesmo tratamento. Entretanto a comparação entre os tratamentos (osmoconcondicionadas e não osmocondicionadas) revela que a partir de CE $12 \mathrm{dS} . \mathrm{m}^{-1}$ as sementes não osmocondicionadas apresentaram a uniformidade do processo germinativo afetada pelas soluções salinas, o que não foi observado nas sementes submetidas ao osmocondicionamento. Vale ressaltar que para as sementes osmocondicionadas, o CUG alcançou um coeficiente máximo de $9,0 \mathrm{sem}_{\text {dia }}{ }^{-1}$ em contraposição aos 3,6 sem.dia ${ }^{-1}$ para as sementes não osmocondicionadas (Tab. 1, Fig. 2).

O tempo médio para sementes osmocondicionadas, tanto para o controle como para concentrações salinas com até CE $12 \mathrm{dS} \mathrm{m}^{-1}$ variou entre 3 a 3,7 dias. A partir da solução com CE $14 \mathrm{dS} \mathrm{m}^{-1}$, observa-se que os picos de germinação passam a ocorrer de 4 a 5 dias. Para as sementes não osmocondicionadas, observa-se que do controle até solução com CE $6 \mathrm{dS} \mathrm{ms}{ }^{-1}$, os picos de germinação apresentam-se de 3,7 a 4,2 dias. Em soluções salinas com CEs 10 e $12 \mathrm{dS} \mathrm{m}^{-1}$, este pico ocorreu entre 4,4 e 4,5, respectivamente. A germinação das sementes não osmocondicionadas apresentou um maior tempo médio à medida que a concentração das soluções salinas foi aumentando, ou seja, houve um efeito direto entre o número de sementes germinadas em função da maior concentração de sal, utilizada no meio para a germinação. Nas concentrações salinas mais elevadas (CEs 14 e 16 dS $\mathrm{m}^{-1}$ ), observou-se um atraso da germinação, em torno de

Tabela 1. Velocidade média $\left(\right.$ dias $\left.^{-1}\right)$, Índice de velocidade de germinação (IVG, sem.dia $\left.{ }^{-1}\right)$ e Coeficiente de uniformidade de germinação $\left(C U G\right.$, sem.dias $\left.{ }^{-2}\right)$ de sementes osmocondicionadas (OS) e não omocondicionadas (NO) de Physalis angulata L. submetidas a diferentes concentrações salinas.

\begin{tabular}{|c|c|c|c|c|c|c|c|c|}
\hline \multirow{2}{*}{$\begin{array}{l}\text { Tipos de } \\
\text { Sementes }\end{array}$} & \multicolumn{8}{|c|}{ Níveis de $\mathrm{NaCl}\left(\mathrm{dS} \mathrm{m}^{-1}\right)$} \\
\hline & 0 & 2 & 4 & 6 & 10 & 12 & 14 & 16 \\
\hline \multicolumn{9}{|c|}{ Velocidade média $\left(\right.$ dias $\left.^{-1}\right)$} \\
\hline OS & $0,2 \mathrm{Aab}$ & $0,3 \mathrm{Aa}$ & $0,3 \mathrm{Aa}$ & $0,3 \mathrm{Aab}$ & $0,3 \mathrm{Aab}$ & $0,3 \mathrm{Aab}$ & $0,2 \mathrm{Abc}$ & $0,2 \mathrm{Ac}$ \\
\hline NO & $0,3 \mathrm{Aa}$ & $0,3 \mathrm{Aa}$ & $0,3 \mathrm{Aa}$ & $0,2 \mathrm{Aab}$ & $0,2 \mathrm{Bab}$ & $0,2 \mathrm{Bab}$ & $0,1 \mathrm{Ab}$ & $0,2 \mathrm{Aab}$ \\
\hline \multicolumn{9}{|c|}{ IVG $\left(\right.$ sem.dia $\left.{ }^{-1}\right)$} \\
\hline OS & 7,2 Aab & $8,0 \mathrm{Aa}$ & $8,0 \mathrm{Aa}$ & 7,2 Aab & 7,1 Aab & $5,9 \mathrm{Ab}$ & $3,2 \mathrm{Ac}$ & $2,2 \mathrm{Ac}$ \\
\hline NO & 6,5 Aab & $6,8 \mathrm{Bb}$ & 7,4 Aa & $5,7 \mathrm{Ab}$ & $3,7 \mathrm{Bc}$ & $0,9 \mathrm{Bd}$ & $0,2 \mathrm{Bd}$ & $0,3 \mathrm{Bd}$ \\
\hline \multicolumn{9}{|c|}{ CUG $\left(\right.$ sem.dias $\left.{ }^{-2}\right)$} \\
\hline OS & $1,4 \mathrm{Ab}$ & $5,3 \mathrm{Ab}$ & $9,0 \mathrm{Aa}$ & $1,0 \mathrm{Ab}$ & $2,3 \mathrm{Ab}$ & $2,6 \mathrm{Ab}$ & $2,1 \mathrm{Ab}$ & $1,4 \mathrm{Ab}$ \\
\hline NO & $2,3 \mathrm{Aa}$ & 3,3 Aa & $3,6 \mathrm{Ba}$ & 2,1 $\mathrm{Aa}$ & $2,1 \mathrm{Aa}$ & $0,7 \mathrm{Bb}$ & $0,0 \mathrm{Bb}$ & $0,2 \mathrm{Bb}$ \\
\hline
\end{tabular}

Médias seguidas de mesma letra, maiúsculas na coluna e minúsculas na linha, não diferem entre si pelo teste de Tukey, ao nível 5\% de probabilidade. 

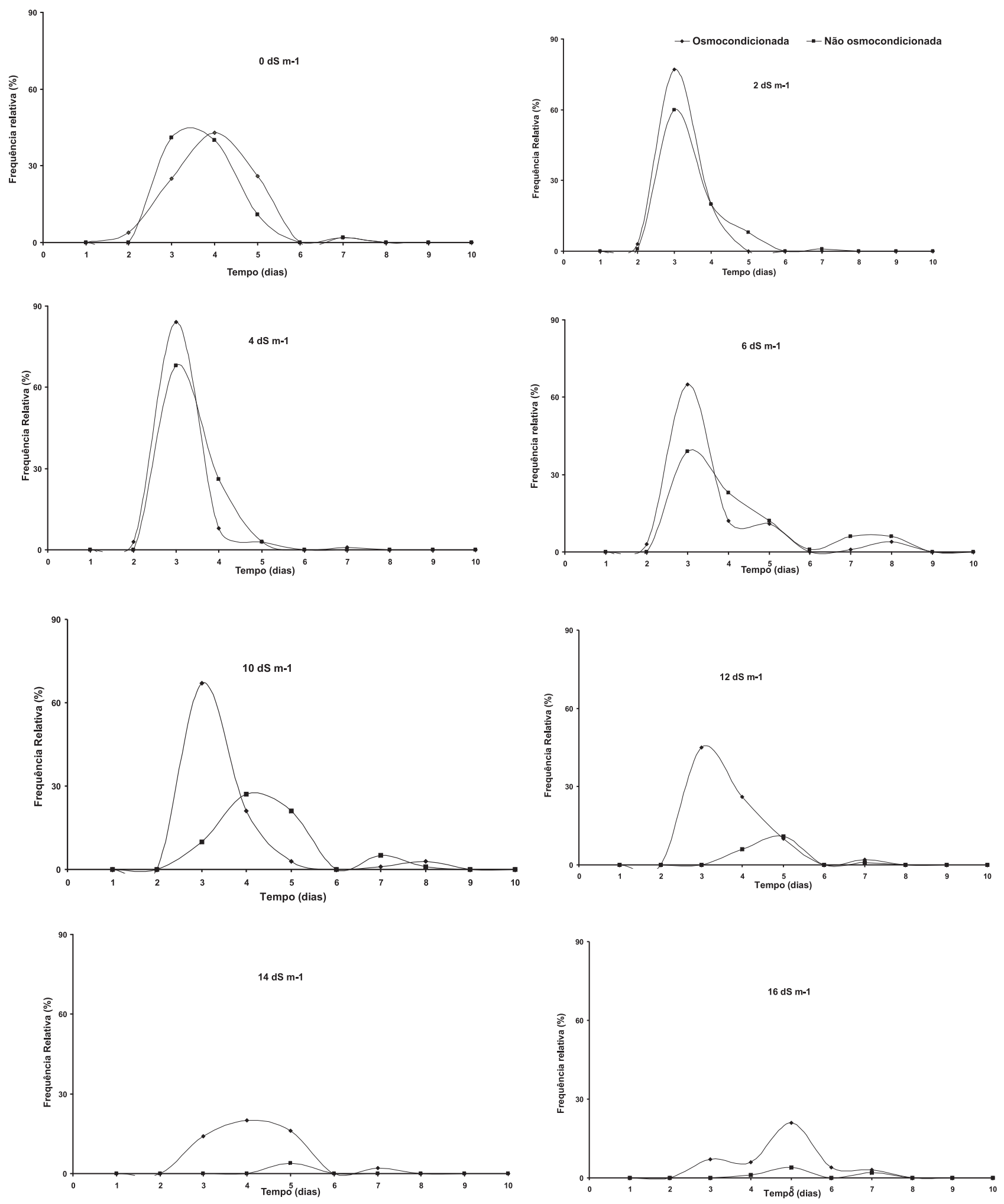

Figura 2. Polígonos de freqüência relativa da germinação de sementes de Physalis angulata L. para diferentes concentrações salinas. OS ( $\bullet$ ) e NO ( 
5 dias, para as sementes não osmocondicionadas (Fig. 2).

O osmocondicionamento mostrou-se eficiente na diminuição do tempo médio de germinação das sementes. No polígono de freqüência relativa fica evidente que sementes osmocondicionadas tiveram um tempo médio de germinação menor que as sementes não osmocondicionadas (Fig 2). Estudos relatam que a principal vantagem do condicionamento das sementes é a maior velocidade de emergência das plântulas favorecendo a maior tolerância das sementes às adversidades climáticas, como deficit hídrico ou ainda excesso de sal no solo, por ocasião da semeadura (Powell 2000; Cantliffe 2003). Segundo Sivritepe et al. (2003) o osmocondicionamento em sementes pode ser adotado como ferramenta tecnológica útil quando se pretende cultivar espécies sob condições de salinidade, e também em ensaios de avaliação de tolerância de espécies a estresse abiótico como a salinidade do meio de cultivo.

Houve uma diminuição progressiva da massa seca de plântulas de Physalis angulata, oriundas de sementes osmocondicionadas e não osmocondicionadas, à medida que ocorreu aumento na concentração das soluções salinas, demonstrado por uma tendência quadrática negativa (Fig. 3). A menor absorção de água pelas sementes atua reduzindo a velocidade dos processos fisiológicos e bioquímicos e, com isso, as plântulas resultantes desse meio, com menor grau de umidade, apresentam menor desenvolvimento, caracterizado pelo menor acúmulo de peso da matéria seca (Rebouças et al. 1989). Observou-se que soluções salinas com CE a partir de $10 \mathrm{dS} \mathrm{m}^{-1}$ levaram ao maior decréscimo na absorção de água pelas sementes acarretando uma redução gradual no peso da massa seca das plântulas, quando comparadas ao controle (CE $0 \mathrm{dS} \mathrm{m}^{-1}$ ). Este resultado evidenciou o efeito prejudicial do incremento de $\mathrm{NaCl}$ no substrato de germinação sobre o crescimento das plântulas de $P$. angulata.

Bosco et al. (2009) observaram que em berinjela (Solanum melongena L.) os tratamentos com menores concentrações de sais proporcionaram maior produção de biomassa. Entretanto, diferenças significativas não foram evidenciadas entre as CEs 1,7 e 6,03 $\mathrm{dS} \mathrm{m}^{-1}$, para a produção de matéria seca de raiz e parte aérea. Esses resultados se devem à possível capacidade dessas plantas se ajustar osmoticamente, acumulando no vacúolo os íons $\mathrm{Na}^{+} \mathrm{e} \mathrm{Cl}$, os quais chegam ao mesofilo foliar (Niu et al. 1995; Ashraf \& Harris 2004), ou sintetizando e acumulando solutos orgânicos que restauram a osmolaridade da célula (Munns \& Tester 2008). O acúmulo desses íons ou solutos permite a manutenção de um gradiente de potencial hídrico ao longo da planta, resultando na absorção e transporte de água até a parte aérea, mesmo que o solo esteja salinizado. As espécies que possuem estes mecanismos são classificadas como moderadamente tolerantes à salinidade (Shannon \& Grieve 1999).

As plântulas de Physalis angulata demonstraram diminuição no crescimento a partir da solução salina com CE de $10 \mathrm{dS} \mathrm{m}^{-1}$, nas quais tanto em plântulas provenientes de sementes osmocondicionadas como não osmocondicionadas, apresentaram $0,24 \mathrm{mg}$ de matéria seca total. Em

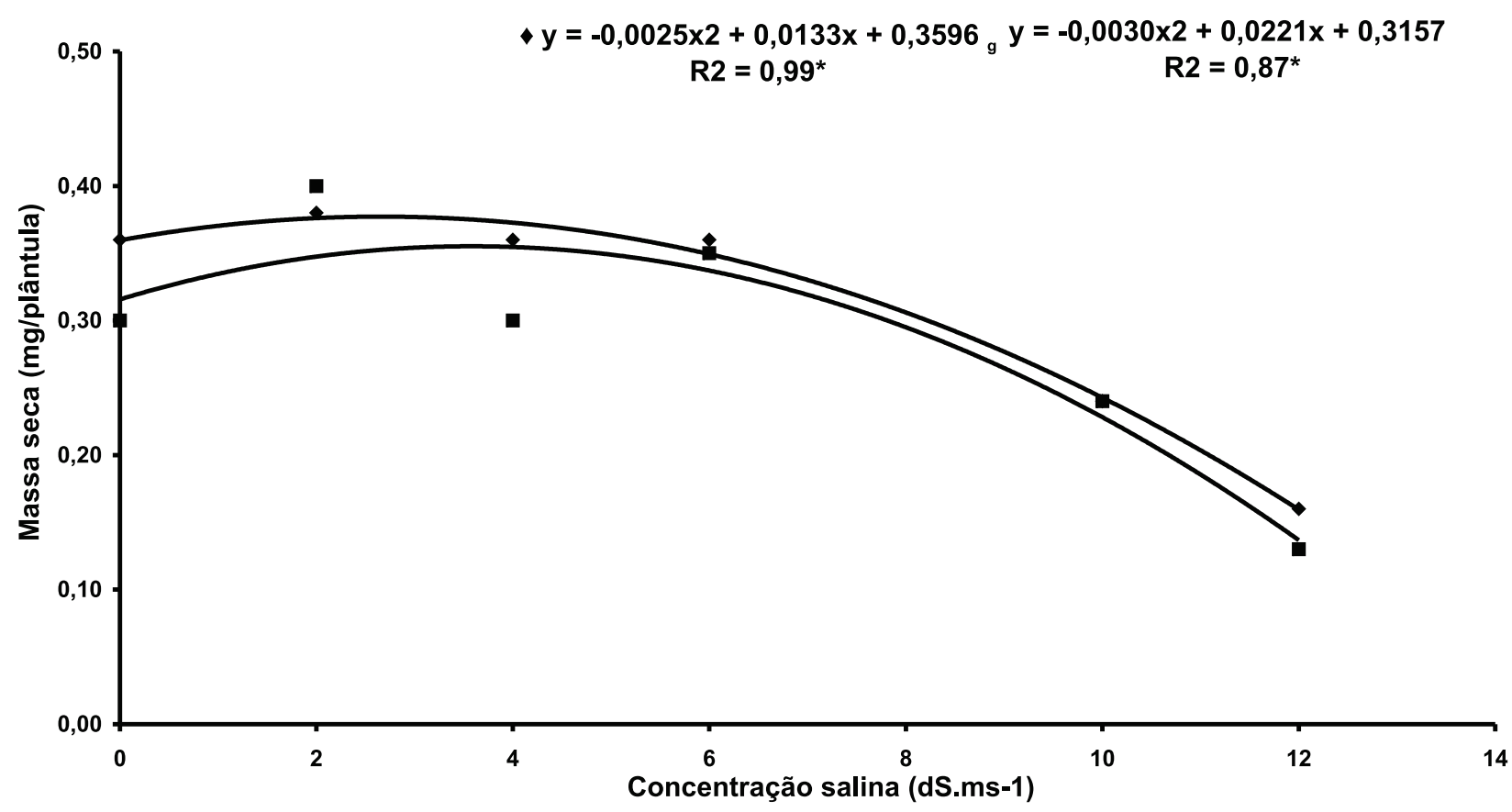

Figura 3. Massa seca total das plântulas de Physalis angulata L. oriundas de sementes osmocondicionadas (OS) e não osmocondicionadas (NO). OS ( ) e NO ( $\mathbf{\square})$. ${ }^{*}$ Significativo a $5 \%$ de probabilidade. 
berinjela, no nível máximo de salinidade (CE de 14,10 dS $\mathrm{m}^{-1}$ ), as reduções de matéria seca foram de: $44,05 \%$ nas raízes e $69,70 \%$ na parte aérea (Bosco et al. 2009). Em Physalis angulata, nessa mesma concentração salina, não foi possível observar desenvolvimento das plântulas em decorrência das anormalidades e efeitos nocivos acentuados, o que impossibilitou a obtenção da matéria seca. Em concentração salina com CE $12 \mathrm{dS} \mathrm{ms}^{-1}$, observou-se uma diminuição acentuada da massa seca tanto de plântulas oriundas de sementes osmocondicionadas $(0,16 \mathrm{mg})$ como não osmocondicionadas $(0,13 \mathrm{mg})$.

A avaliação da sensibilidade das plantas ao estresse salino é freqüentemente baseada nos dados de crescimento da planta (Liu \& Staden 2001). Em 14 cultivares de batata (Solanum tuberosum L.) irrigada com águas salinas $\left(6,1 \mathrm{a} 6,7 \mathrm{dS} \mathrm{m}^{-1}\right)$ foi demonstrado que a batata é classificada como moderadamente tolerante à salinidade (Levy 1992). A explicação mais aceita para a inibição do crescimento pelo sal é a redução do potencial osmótico da solução de cultivo, podendo também ocasionar toxicidade iônica, desequilíbrios nutricionais ou ambos, devido à acumulação excessiva de certos íons nos tecidos vegetais. Além disso, as plantas fecham os estômatos para reduzir as perdas de água por transpiração, resultando em uma menor taxa fotossintética, o que constitui uma das causas do crescimento reduzido das espécies sob condições de estresse salino (Flowers 2004; Navarro et al. 2007; Munns $\&$ Tester, 2008).

Apesar da afirmação de que o osmocondicionamento possa ser eficaz nas primeiras fases do desenvolvimento das plântulas (Gray et al. 1991; Khan et al. 2009), vale ressaltar que neste trabalho, este fato não foi observado. Ficou evidente que tanto em plântulas provenientes de sementes osmocondicionadas como não osmocondicionadas ocorreu uma tendência semelhante de diminuição da massa seca, apesar da taxa de germinação ter sido mais elevada em sementes osmocondicionadas._

\section{Agradecimentos}

A primeira autora agradece a FAPESB, pela concessão da bolsa de Doutorado e ao Programa de Pós-Graduação em Biotecnologia da Universidade Estadual de Feira de Santana, a segunda autora agradece ao CNPq, pela concessão da bolsa DTI.

\section{Referências bibliográficas}

Abreu, C.E.B.; Prisco, J.T.; Nogueira, A.R.C.; Bezerra, M.A.; Lacerda C.F. \& Gomes-Filho, E. 2008. Physiological and biochemical changes occurring in dwarf-cashew seedlings subjected to salt stress. Brazilian Journal of Plant Physiology 20(2):105-118.

Ashraf, M. \& Foolad, M. R. 2005. Pre-sowing seed treatment-a shotgun approach to improve germination growth and crop yield under saline and none-saline conditions. Advances in Agronomy 88: 223-271.
Ashraf, M. \& Harris, P.J.C. 2004. Potential biochemical indicators of salinity tolerance in plants. Plant Science 166:3-16.

Anwar, A.K.; Kar-Ling,T.; Knypl, J.S.; Borkonska, B. \& Loy, E.P. 1978. Osmotic conditioning of seed: physiological and biochemical changes. Acta Horticulturae 1(83): 267-278.

Bewley, J.D. \& Black, M. 1994. Seeds: physiology of development and germination. New York, Plenum Press.

Borges, E.E.L.; Perez, S.C.J.G.; Borges, R.C.G.; Rezende, S.T. \& Garcia, S.R. 2002. Comportamento fisiológico de sementes osmocondicionadas de Platymiscium pubescens Micheli (Tamboril-da-Mata). Revista Árvore 26(5): 603-613.

Bosco, M.R. de O.; Oliveira, A.A.B.; Hernandez, F.F.F \& Lacerda, C.F. 2009. Efeito do $\mathrm{NaCl}$ sobre o crescimento, fotossíntese e relações hídricas de plantas de berinjela. Revista Ceres 56(3): 296-302.

Bradford, K. J. 1986. Manipulation of seed water relations via osmotic priming to improve germination under stress conditions. HortScience 21(5): 1105-1112.

Brocklehurst, P.A. \& Dearman, J. 1983. Interactions between seed priming treatments and nine seed lots of carrot, celery and onion. I. Annals of Applied Biology 102(3): 577-584.

Cantliffe, D.J. 2003. Seed Enhancements. Acta Horticulturae 607: 53-59.

Chiang, H.C.; Jaw, S.M. \& Chen, C.F. 1992. Effects of Physalin B and Physalin F on Various Human Leukemia Cells in vitro. Anticancer Research 12:1155-1162.

D'Arcy, W.; Rojas, C.B. \& N.E.E, M.H. 2005. Solanaceae. Flora of the Venezuelas Guyana 9: 194-246.

Ferreira, D.F. 2000. Análises estatísticas por meio do Sisvar para Windows versão 4.0. Pp. 255-258. In: Anais da Reunião Anual da Região Brasileira da Sociedade Internacional de Biometria. 2000. São Carlos, UFSCar.

Ferreira, P.A. 1997. Aspectos físico-químicos do solo. Pp. 37-67. In: Gheyi, H.R.; Queiroz, J.E. \& Medeiros, J.F. Manejo e controle da salinidade na agricultura irrigada. Campina Grande, UFPB/SBEA.

Flowers, T.J. 2004. Improving crop salt tolerance. Journal of Experimental Botany 55:307-319.

Flors, V.; Paradís, M.; García-Andrade, J.; Cerezo, M.; Bosch, G.C. \& Pilar, G.A.P. 2007. A tolerant behavior in salt-sensitive tomato plants can be mimicked by chemical stimuli. Plant Signaling \& Behavior 2(1): 50-57.

Gray, D.; Steckel, J.R.A.; Drew, R.L.K. \& Keefe, P.D. 1991. The contribution of seed characters to carrot plant and root size variability. Seed Science and Technology 19(3): 655-664.

Heuer, B. Photosynthetic carbon metabolism of crops under salt stress. In: Pessarakli, M. (Ed.). Handbook of photosynthesis. New York, Marcel Dekker.

Khan, H.A.; Ayub, C.M.; Pervez, M.A.; Bilal, R.M., Shahid, M.A. \& Ziaf, K. 2009. Effect of seed priming with $\mathrm{NaCl}$ on salinity tolerance of hot pepper (Capsicum annuum L.) at seedling stage. Soil \& Environment 28(1): 81-87.

Larcher, W. 2000. Ecofisiologia vegetal. São Carlos, Rima.

Levy, D. 1992. The response of potatoes (Solanum tuberosum L.) to salinity: plant growth and tuber yields in the arid desert of Israel. Annals of Applied Biology 120: 547-555.

Lin, Y.S.; Chiang, H.S.; Hone, E.; Shin, S.J. \& Won, M.H. 1992. Immunomodulatory activity of various fractions derived from Physalis angulata L. extract. American Journal of Chinese Medicine 20: 233-243.

Liu, T. \& Staden, J. 2001. Growth rate, water relations and ion accumulation of soybean callus lines differing in salinity tolerance under salinity stress and its subsequent relief. Plant Growth Regulation 34: 277-285.

Matos, F.J.A. 2000. Plantas Medicinais: guia de seleção e emprego de plantas usadas em fitoterapia no nordeste do Brasil. 2 ed. Fortaleza, UFC.

Mohammadi, G.R. 2009. The influence of $\mathrm{NaCl}$ priming on seed germination and seedling growth of canola (Brassica napus L.) under salinity conditions. American-Eurasian Journal of Agricultural \& Environmental Sciences 5(5): 696-700.

Munns, R. \& Tester, M. 2008. Mechanisms of salinity tolerance. Annual Review Plant Biology 59:651-681. 
Nascimento, W.M. 1998. Condicionamento osmótico de sementes de hortaliças: potencialidades e implicações. Horticultura Brasileira 16(2): 106-109.

Nakagawa, J. 1999. Testes de vigor baseados no desempenho das plântulas. Pp. 2-24. In: Krzyzanowsky, F.C.; Vieira, R.D.\& França Neto, J.B. (Eds.). Vigor de sementes: conceitos e testes. Londrina, ABRATES.

Navarro, A.; Bañon, S.; Olmos, E.; \& Sánchez-Blanco, M.J. 2007. Effects of sodium chloride on water potential components, hydraulic conductivity, gas exchange and leaf ultrastructure of Arbutus unedo plants. Plant Science 172: 473-480.

Niu, X., Bressan, R.A., Hasegawa, P.M. \& Pardo, J.M. 1995. Ion homeostasis in $\mathrm{NaCl}$ stress environments. Plant Physiology 109: 735-742.

Passam, H.C.; Karavites, P.I.; Papandreou, A.A.; Thanos, C.A. \& Georghiou, K. 1986. Osmocondictionig of seeds in relation to growth and fruit yield of aubergine, peper, cucumber and melon in unheated greenhouse cultivation. Scientia Horticulturae 38: 207-216.

Powell, A.A., Yule, L.J., Jingh, H.C. \& Groots, P.C. 2000. The influence of aerated hydration seed treatment on seed longevity as assessed by the viability equation. Journal of Experimental Botany 51: 2031-2043.

Queiroga, R.C.F; Andrade Neto, R.C; Nunes, G.H.S; Medeiros, J.F \& Araújo, W.B.M. 2006. Germinação e crescimento inicial de híbridos de meloeiro em função da salinidade. Horticultura Brasileira 24: 315-319.

Rebouças, M.A.; Façanha, J.G.V.; Ferreira, L.G.R. \& Prisco, J.T. 1989. Crescimento e conteúdo de N, P, Ke Na em três cultivares de algodão sob condições de estresse salino. Revista Brasileira de Fisiologia Vegetal 1(1): 79-85.
Shannon, M.C. \& Grieve, C.M. 1999. Tolerance of vegetable crops to salinity. Scientia Horticulturae 78(1-4): 5-38.

Sivritepe, N.; Sivritepe, H.O. \& Eris, A. 2003. The effect of $\mathrm{NaCl}$ priming on salt tolerance in melon seedling grown under saline conditions. Scientae Horticulturae 97: 229-237.

Silva, M.J.; Souza, J.G.; Barreiro-Neto, M. \& Silva, J.V. 1992. Seleção de três cultivares de algodoeiro para tolerância à germinação em condições salinas. Pesquisa Agropecuária Brasileira 27(9): 1311-1317.

Silva, D. \& Pruski, F.F. 1997. Recursos hídricos e desenvolvimento sustentável da agricultura. Brasília, MMA, SBH, ABEAS.

Souza, C.L.M. 2009. Germinabilidade de sementes de Physalis angulata L. (Solanaceae) sob condições de estresses abióticos. Dissertação de Mestrado. Universidade Estadual de Feira de Santana, Feira de Santana..

Tomassini, T.C.B.; Barbi, N.; Ribeiro, I.M. \& Xavier, D.C.D. 2000. Gênero Physalis - uma revisão sobre vitaesteróides. Química Nova 23(1): 47-57.

Villela, F.A.; Doni Filho, L.D \& Sequeira, E.L. 1991. Tabela de potencial osmótico em função da concentração de polietilenoglicol 6.000 e da temperatura. Pesquisa Agropecuária Brasileira 26(11/12): 1957-1968.

Warren, J.E. \& Bennett, M.A. 1997. Seed hydration using the drum priming system. HortScience 32(7): 1220-1221.

Wien, H.C. 1997. The physiology of vegetable crops. Peppers. New York, Cab International.

Yokoi, S.; Bressan, R.A. \& Hasegawa, P.M. 2002. Salt stress tolerance of plants. Jircas Working Report 23: 25-33.

Zhu, J.K. 2002. Salt and drought stress signal transduction in plants. Annual Review of Plant Biology 53: 247-273 